\title{
The Coxalgic Pelvis.
}

\section{An Obstetrical Enguiry into the Lateral Tilting of the PELVIS.}

\author{
By Henry Briggs, M.B., F.R.C.S.,
} Professor of Obstetrics and Gynacology, University of Liverpool.

The feature of the coxalgic pelvis is its asymmetry; almost entirely due to alterations in the innominate bones; commonly the product of unilateral hip-joint disease with ankylosis in childhood; and occasionally the cause of a severe dystocia in the adult woman at, or about, the full term of pregnancy.

'Th' deformity in one-fifth of Prouvost's 50 cases was sufficient to interferc, more or less seriously, with labour.

The photographs (A) and (B) reproduce the distorted physical features of the coxalgic pelvis on the diseased side, the stunted and thinned innominate bone has acquired an exaggerated outward bend and is considerably contracted. On the healthy side, the more fully grown innominate bone, by weight carrying, is flattened and its accommodation is less curtailed. The oblique pelvic contraction extends to the outlet where the tuber ischii on the diseased side is further from the middle line and on the sound side nearer to the middle line. The sacro-iliac joints, as correctly stated by $\mathrm{H}$. Peters, are not affected. The sacral alæe retain their symmetry, hence the deformity of the coxalgic pelvis is the reverse of that of the Nægele pelvis.

For the extreme example of coxalgic pelvis in $(A)$ the Liverpool University is indebted to Professor Thomas Wilson, of Birmingham, who lent the original with permission for the plaster of paris copy, made in Liverpool by an Italian moulder.

Concerning the plaster of paris copy, its present pose (C) on its museum mountings has attracted obstetrical enquiry.

Has the pelvis been correctly posed? And, more particularly, ought the pelvis to be relatively raised or lowered on the diseased side?

The first mounting, after the custom of the anatomical mechanician, displayed the two halves of the pelvis on an approximate level.

Local obstetrical experience had planned the raising of the diseased side.

Local surgical guidance was sought, with the result that the pelvis was lowered on the diseased side.

Against this surgical verdict obstetrical appeal was renewed on clinical evidence, supported bv radiography and photography.

Two women, both primigravidæ, each with ankylosis of the left hip joint from childhood, had been recently in labour: (r) aged 26 ; at the full term; forceps failed; the protracted labour was completed by craniotomy; (2) aged 35 ; the birth of a healthy, living, 


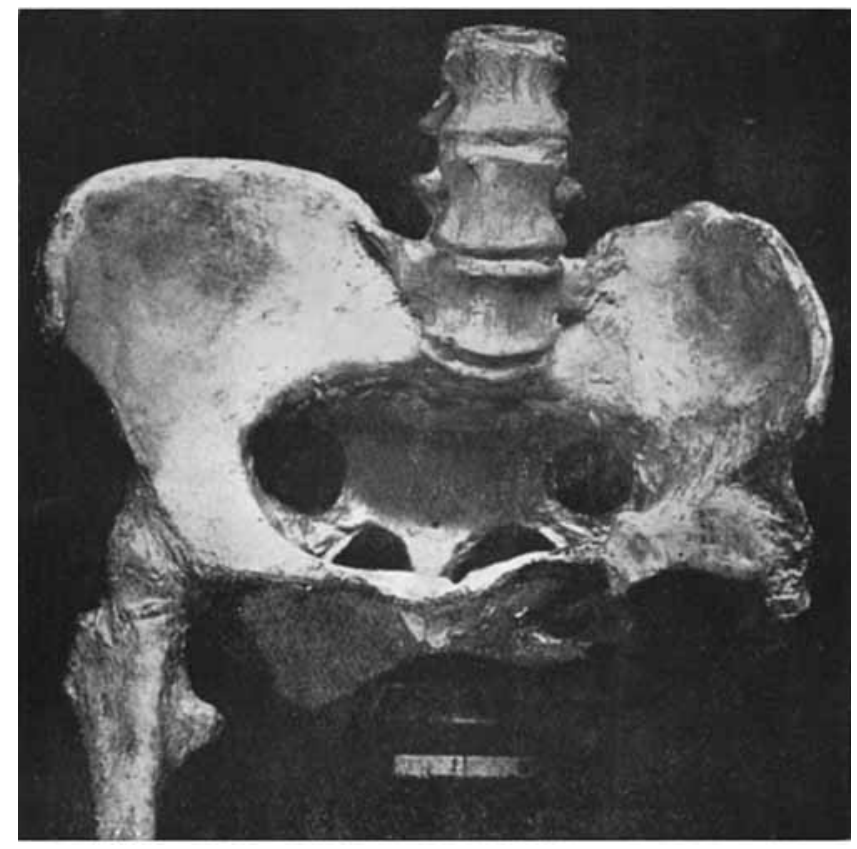

(A) More asymmetry; unilateral hip disease; common form.

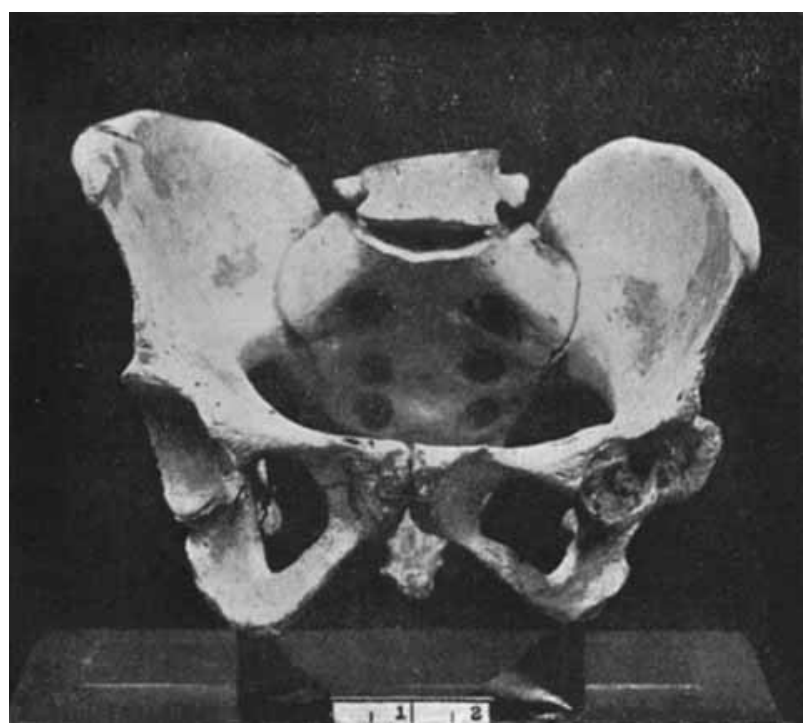

(B) Less asymmetry; bilateral hip disease; rare form. Severe pelvic contraction in both $\mathrm{A}$ and $\mathrm{B}$. 


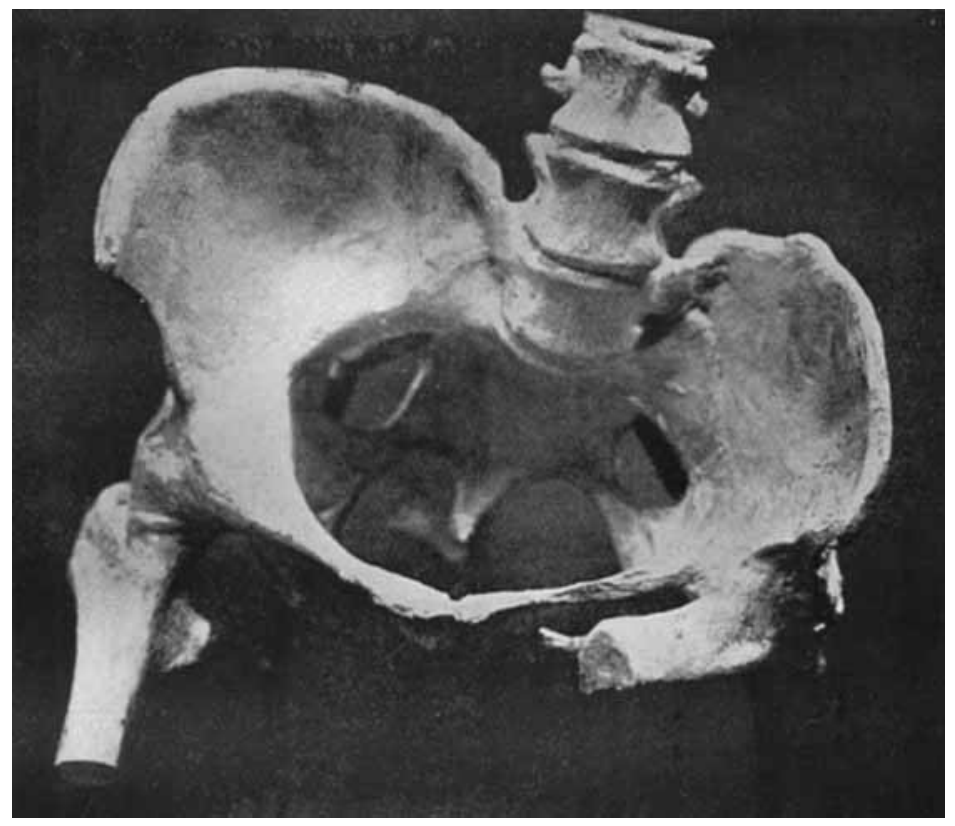

(C) Its pose for obstetrical teaching? 


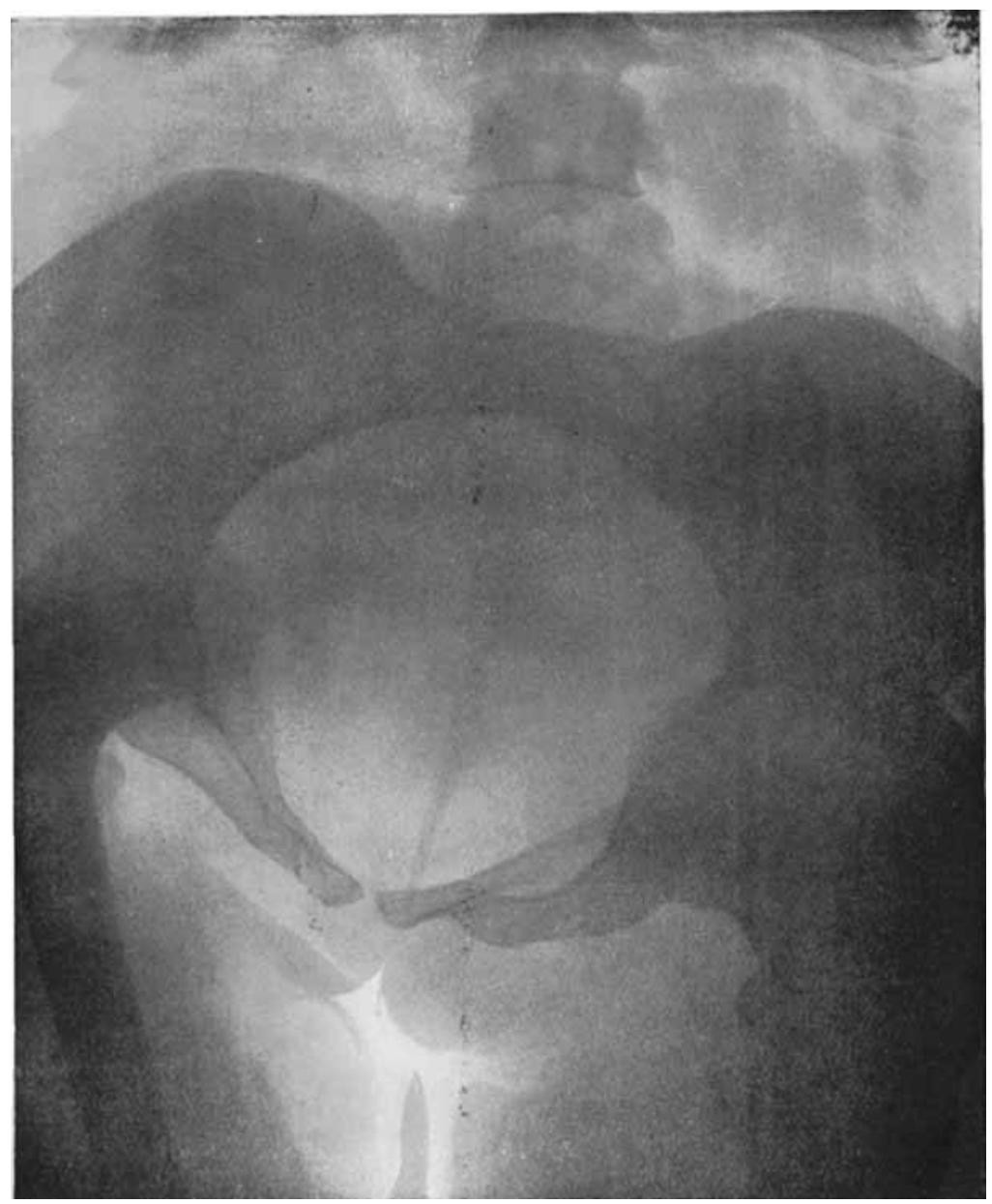

Mrs. W. x-my by Wr. Thustan Holland; back view; left hip raised. 


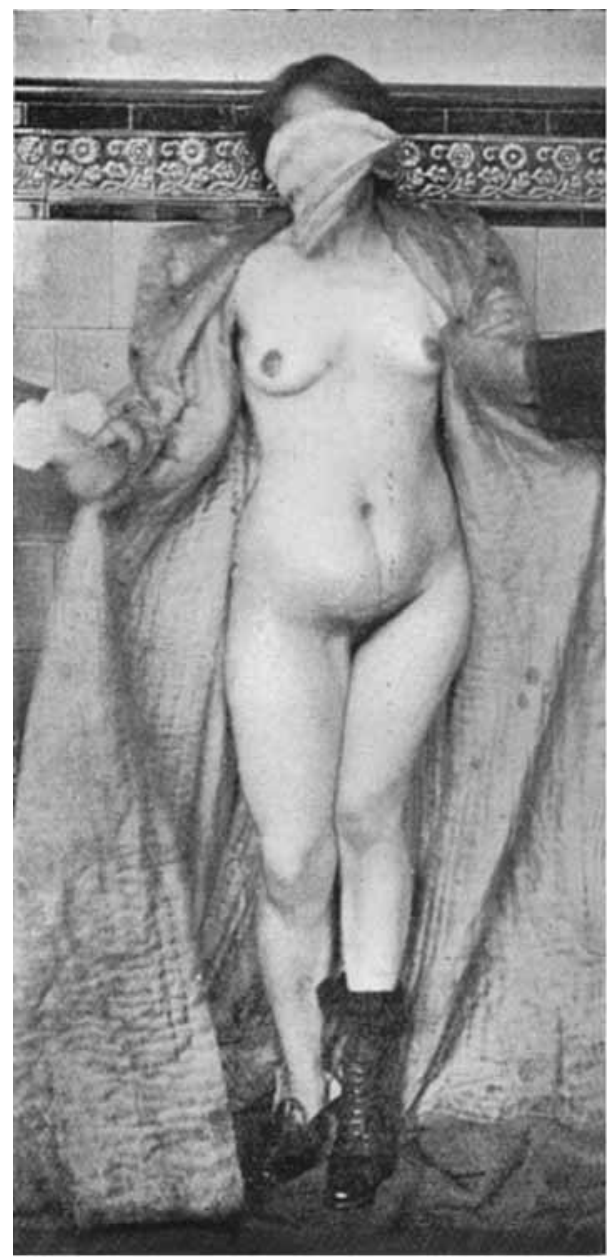

Mrs. W.

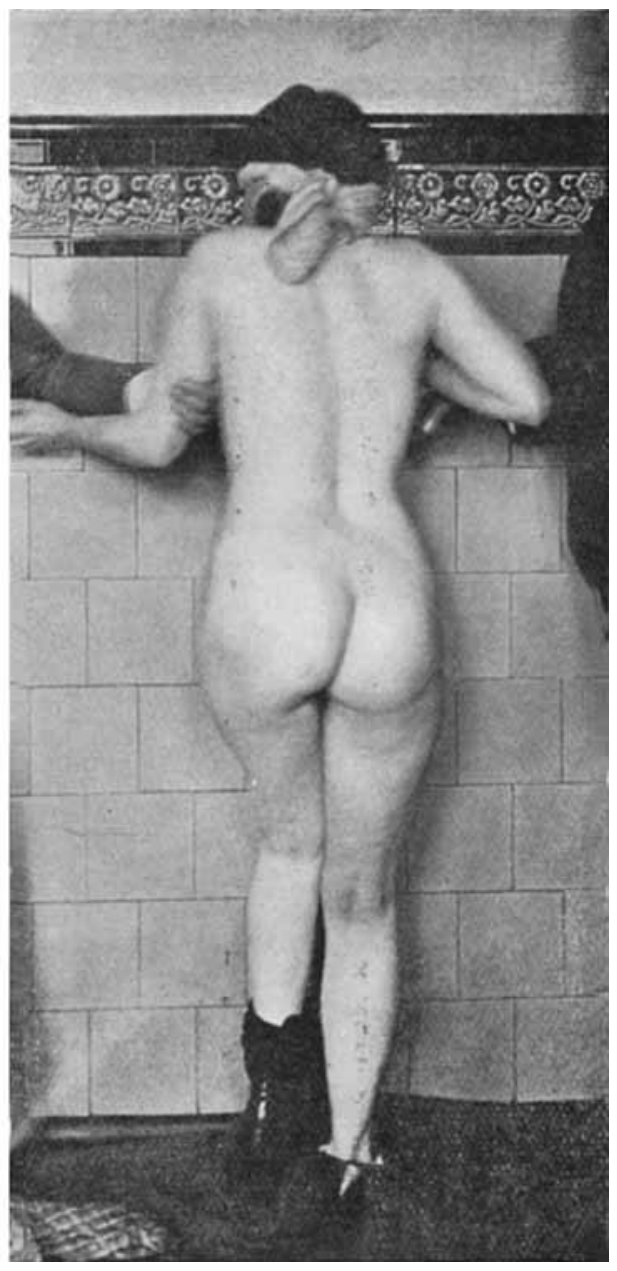

Mrs. W.

Left hip considerably raised. 
male child, followed the induction of premature labour and the use of forceps in the 36 th week of pregnancy.

Brief notes of these two cases, with photographs, are appended.

\section{(i) Severe Dystocia at the Full Term.}

Mrs. W., aged 26, a primigravida; in labour at home; head arrested at the brim; forceps extraction failed; craniotomy May 26th, I9I4.

Above the obliquely contracted pelvis the head was lying in the R.O.A. position, with inadequate flexion, as shown by the forward site of the perforation of the left parietal bone adjacent to the coronal suture. The weight of the fœtus, without cranial contents, $4 \mathrm{lb}$. I $5 \mathrm{oz}$.

The height of the patient 5 feet; the left hip joint ankylosed; the pelvis considerably raised on the diseased side.

The pelvic measurements: intercristal, $9 \frac{3}{4}$; interspinous, $9 \frac{3}{4}$; external conjugate, 7 .

\section{(2) A Mild Degree of Deformity.}

M. E., aged 35, a primigravida, in the 36 th week of pregnancy. Labour was induced. Two gum elastic bougies (Nos. 9 and ro) were placed. At the end of 46 hours the bougies were removed. At the end of $75 \frac{1}{2}$ hours the head, in the R.O.A. position, became impacted in the pelvic cavity. An anæsthetic was necessary to catheterise the full bladder. There were considerable head moulding and a moderate caput. Forceps extraction required a moderately hard pull. A living male child, $5 \mathrm{lb} .9 \mathrm{oz}$. in weight, I7 inches long. September 6th, I9r4.

The patient's height 5 feet 4 inches; the left hip joint ankylosed; the left side of the pelvis slightly raised.

The pelvic measurements: intercristal, Io $\frac{1}{2}$; interspinous, $9 \frac{1}{2}$; external conjugate, $7 \frac{3}{4}$.

\section{(3) A Mild Degree of Deformity.}

(3) M. M., aged 23, a primigravida, who refused to be photographed, was admitted into the Maternity Hospital in labour on October I2, I9I4, with a contracted coxalgic pelvis and an ankylosed raised left hip. The vertex presented in the R.O.A. position, and labour ended naturally.

In these instances the evidence is unassailable that the diseased is carried higher than the sound side, whether the degree of deformity be (1) severe or (2) and (3) mild.

The mechanism of labour in each was consistent with the upward lateral tilting of the more contracted left half of the pelvis, and the R.O.A. position of the vertex was regulated by the wider occiput of the flexed head accommodating itself in the more roomy right half of the pelvis.

Two omissions at this stage of the enquiry are conspicuous : (I) a statement of the mechanism of labour when the lateral pelvic tilting lowers the contracted side; (2) a reference to multiparous cases. 
In supplying the first omission-the mechanism of labour in asymmetrical distortion of the pelvis laterally lowered on the contracted side-it is not in the coxalgic pelvis that the opportunity lies, but in an allied asymmetrical distortion, the consequence of shortening of the lower limb early in life.

R'obert Barnes, ${ }^{*}$ in 1860 , described a case of asymmetrical distortion of the pelvis, the result of unequal length of the legs. In the patient there was shortening and lameness of the left leg from a fractured ankle at the age of two years. At the age of 20, in the patient's first labour, craniotomy was performed. At the age of 21 , in the second labour, a smaller child was extracted with difficulty by forceps.

Barnes, who attended the labours, reported the lowering of the contracted left side of the pelvis and the larger occipital end of the flexed fotal head, loosely lying over the pelvic brim on that side, in the L.O.A. position.

Barnes discussed version as a means of bringing the larger occiput into the larger half of the pelvic brim.

In supplying the second omission by a reference to two multiparous cases of coxalgic pelvis, each tilted laterally upwards on the contracted left side of the pelvis, only recent labour reports are available.

E. S., aged 29, a iv-para. All natural labours at the full term. Fifth pregnancy. Labour at the full term. R.O.A. position of the vertex. Spontaneous delivery October 23rd, 19I4.

Height of patient 4 feet 8 inches. Excision of the left hip at the age of seven years. Fibrous ankylosis.

M. P., aged 37 , iv-para. The fourth labour. R.O.P. position of vertex. Forceps delivery June 3 rd, I9I 2 .

Fifth labour, L.O.A. position of vertex. Forceps October 26th, I9I4.

Height 5 feet $\mathrm{I}$ inch. A hip splint was worn between the ages of 3 and 13 years.

The intrinsic alterations in the coxalgic pelvis have been exhaustively described by obstetrical authorities-Tarnier and Budin, Paul Bar, Breus and Kolisko, Von Winckel, and others.

But the lateral pelvic tilting has almost invariably escaped allusion or description. side.

Whitridge Williams describes the tilting upwards of the well

De Lee, on the contrary, states that the pelvis is raised on the diseased side.

The obliquity of the rhomboid of Michaelis carties a two-fold causation: (I) the defective development and (2) the tilting of the ilium upwards on the diseased side. The latter alone has apparently appealed to De Lee.

* Obst. Soc. Trans. Vol. II. 1861. 


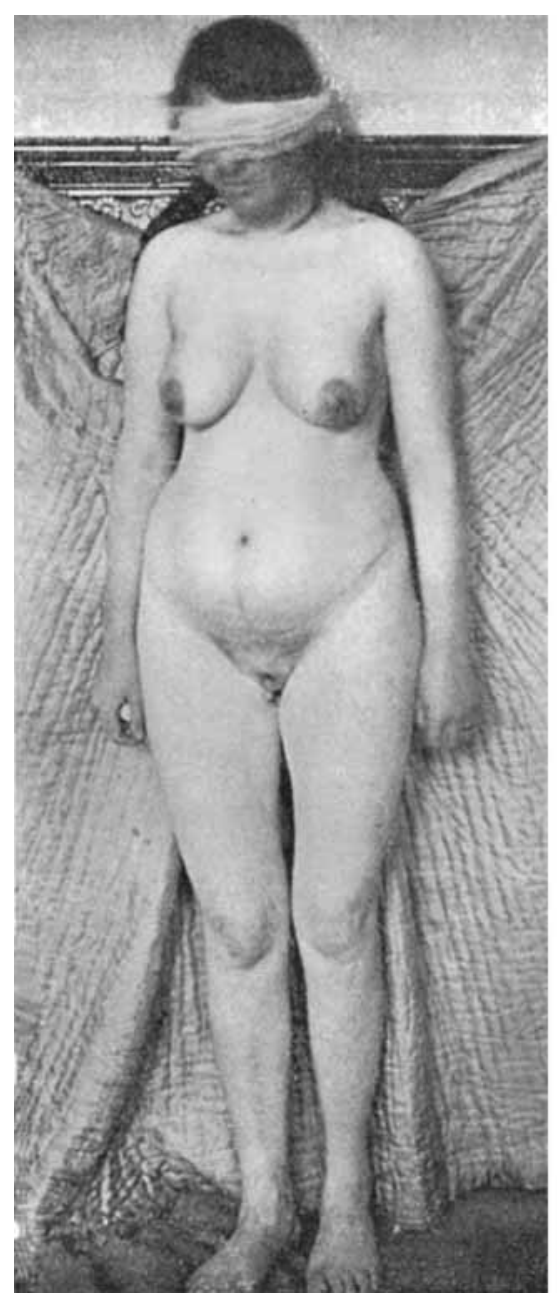

M. E.

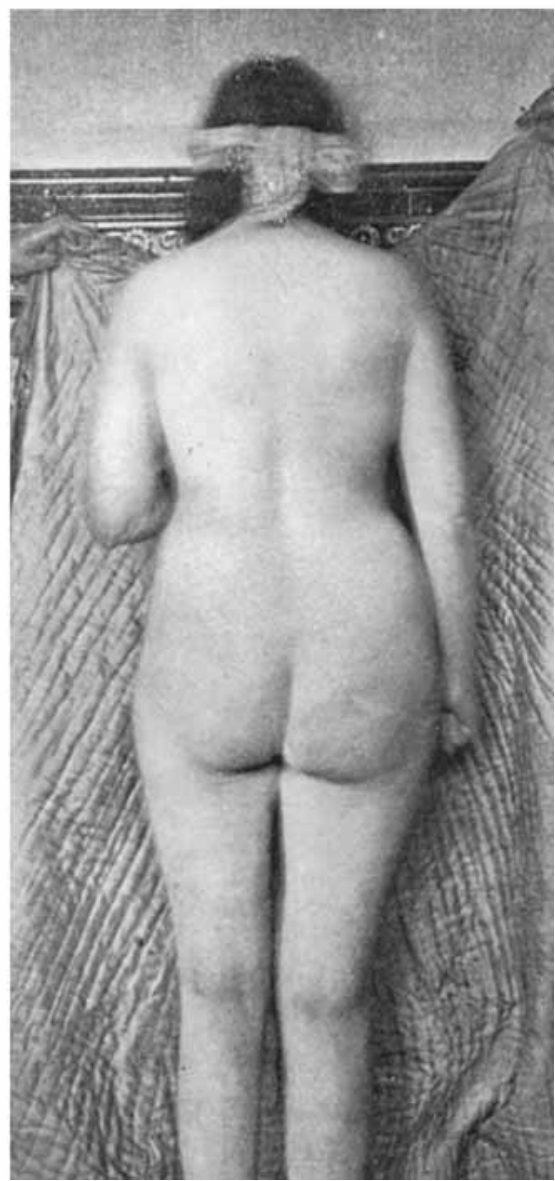

M. E.

Left hip distinctly raised. 


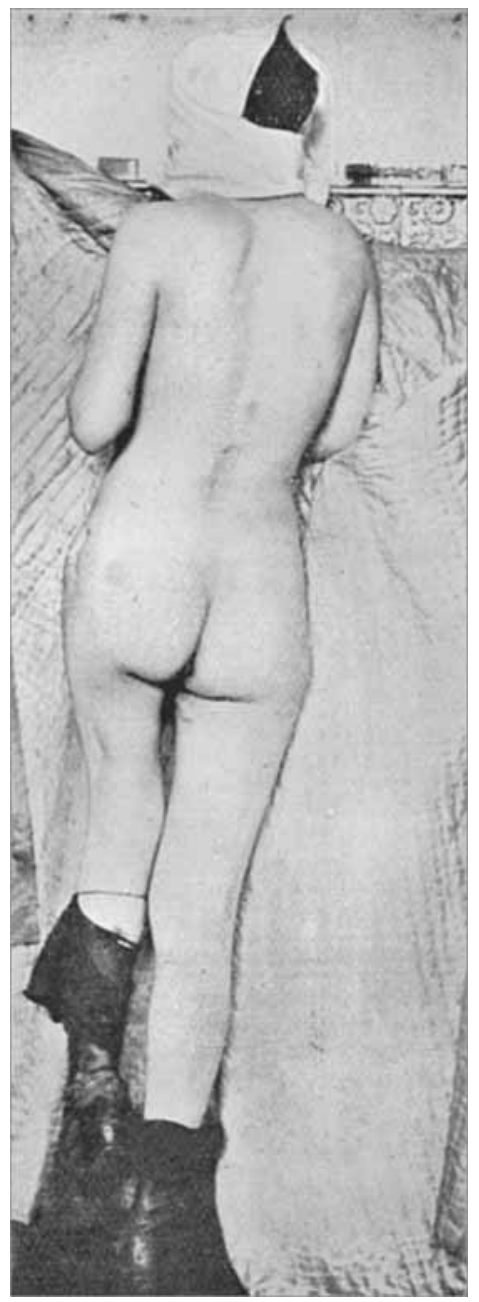

E. S.

Left hip considerably raised. 


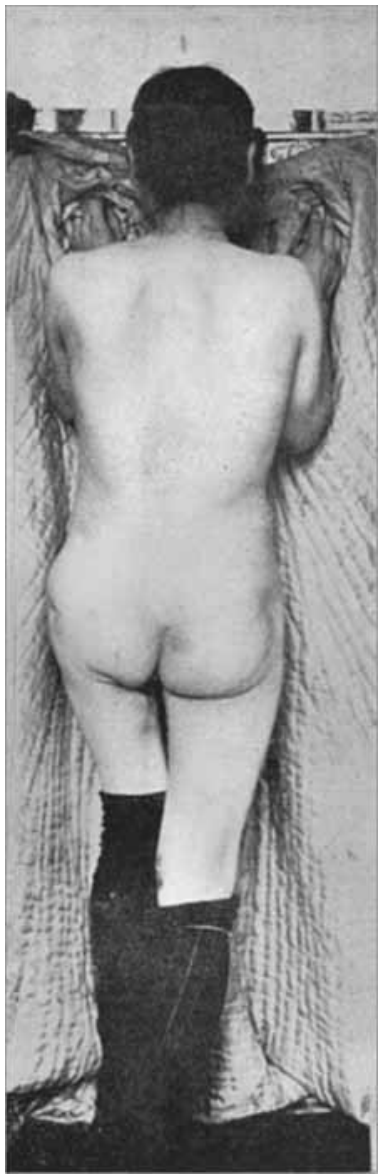

M. P.

Left hip considerably raised. 
Summary of Clinical Evidence.

Five patients, all with lateral tilting of the pelvis. In each the left half of the pelvis, the diseased side, was raised. In four R.O.A. and in one L.O.A. were recorded as the positions of the vertex presentations.

Natural delivery in two, forceps in one, induction in one, craniotomy in one.

The conclusion is inevitable that the diseased side is raised and that the mechanism of labour is thereby favourably influenced in the moderately contracted coxalgic pelvis. 